\title{
Cross-band versus intra-band pairing in superconductors: signatures and consequences of the interplay
}

\author{
A. A. Vargas-Paredes, ${ }^{1,2}$ A. A. Shanenko, ${ }^{3}$ A. Vagov, ${ }^{4}$ M. V. Milošević, ${ }^{2,1}$ and A. Perali ${ }^{1}$ \\ ${ }^{1}$ School of Pharmacy, Physics Unit, Università di Camerino, 62032 Camerino, Italy \\ ${ }^{2}$ Department of Physics, University of Antwerp, Groenenborgerlaan 171, B-2020 Antwerp, Belgium \\ ${ }^{3}$ Universidade Federal de Pernambuco, Av. Prof. Luiz Freire, s/n, 50670-901 Recife-PE, Brazil \\ ${ }^{4}$ Institut für Theoretische Physik III, Bayreuth Universität, Bayreuth, D-95440, Germany
}

(Dated: August 27, 2021)

\begin{abstract}
We analyze the paradigmatic competition between intra-band and cross-band Cooper-pair formation in two-band superconductors, neglected in most works to date. We derive the phase-sensitive gap equations and describe the crossover between the intraband-dominated and the crossbanddominated regimes, delimited by a "gapless" state. Experimental signatures of crosspairing comprise notable gap-splitting in the excitation spectrum, non-BCS behavior of gaps versus temperature, as well as changes in the pairing symmetry as a function of temperature. The consequences of these findings are illustrated on the examples of $\mathrm{MgB}_{2}$ and $\mathrm{Ba}_{0.6} \mathrm{~K}_{0.4} \mathrm{Fe}_{2} \mathrm{As}_{2}$.
\end{abstract}

Multiband superconductivity is known to promote novel quantum phenomena of great fundamental importance and versatility [1. Among recent examples are optically excited collective modes in multiband $\mathrm{MgB}_{2}$ [2], the emergent phenomena at the BCS-BEC crossover in FeSe [3], and at oxide intefaces [4. Strong scientific appeal of multiband superconductivity stems from its pronounced tunability. External pressure, lattice strain effects, gating, chemical doping, photo-induction, quantum confinement and surface effects are all able to move and change the band dispersions and the position of the chemical potential with respect to Lifshitz transitions [4 9], where superconducting properties can radically change.

To date, multiband electronic structure is proven to be of crucial importance in rather versatile superconducting systems, such as $\mathrm{MgB}_{2}$ [10], iron-based compounds [11[16], superconducting nanostructures [17 21], 2D electron gases at interfaces 22 24, metal-organic superconductors 25-27, etc. In such multiband superconductors, the pairing interaction and the proximity/hybridization of two or more bands can result in the formation of Cooper pairs with electrons originating from different bands, a phenomenon termed "cross-band pairing" or simply "crosspairing". This pairing is to be distinguished from the Josephson-like pair transfer between the intraband condensates, which is usually taken as their sole coupling in multiband superconductors. Crosspairing and intraband pairing are intuitively competitive, therefore it is necessary to understand their interplay qualitatively and quantitatively, together with associated changes in physical properties and observables. Such understanding is far from established, as crosspairing and its competition with intraband pairing were predominantly neglected in the studies to date. In superfluid systems with at least two fermionic species, the partially overlapping bands at the Fermi level are prone to crosspairing, as discussed in Refs. 28 and 29, In superconductors, the hybridization of multiple bands close to the Fermi level is favorable for cross-band pair formation. This occurs

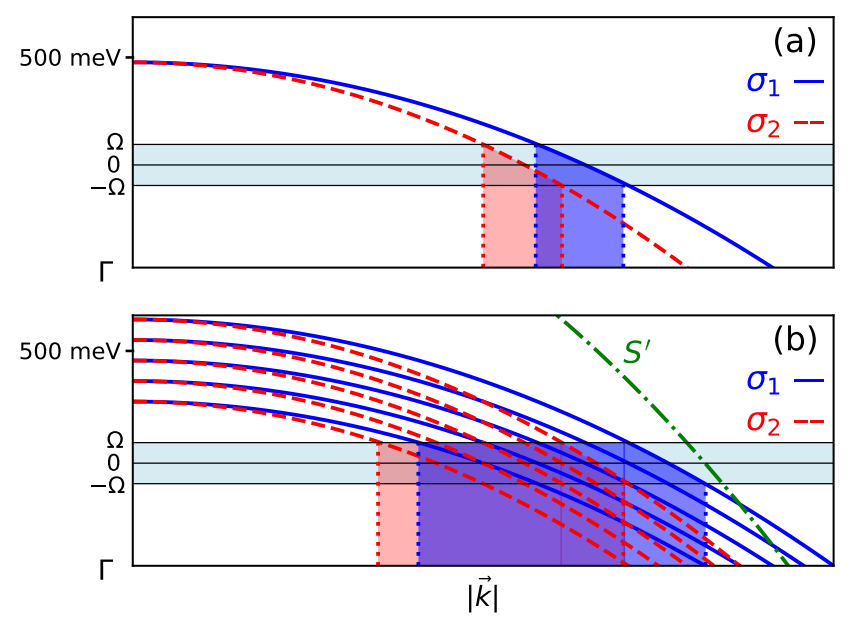

FIG. 1. The relevance of crosspairing is illustrated based on the band structure of (a) bulk $\mathrm{MgB}_{2}$ [35] and (b) 6-monolayer $\mathrm{MgB}_{2}$ [36]. Only $\sigma$ bands close to the $\Gamma$ point of the Brillouine zone are shown, with chemical potential $\mu=500 \mathrm{meV}$ and energy scale of the pairing $\Omega=75 \mathrm{meV}$. In (b), each interior monolayer contributes a pair of hole-like bands $\sigma_{1}$ and $\sigma_{2}$, and the surface band is denoted by $S^{\prime}$. The (purple) overlapping shadows project the momentum states where cross-band pairing between opposite momenta states among the $\sigma$ bands is feasible.

in the iron-based superconductors (FeSCs) which present hybridized orbitals [30, 31], cuprates with the hybridization of $d_{x^{2}-y^{2}}$ and $d_{z^{2}}$ orbitals [32, 33, and also in the heavy-fermion compounds, where crosspairing between electrons with $f$ and $d$ orbital character has been considered [34. However, even without hybridization, the plain proximity of multiple bands can facilitate crosspairing, as illustrated in Fig. 1 for bulk and atomically-thin $\mathrm{MgB}_{2}$.

In this Letter, we examine the interplay between intraand cross-band pairing in two-band superconductors and its experimental signatures. We reformulate the mean- 
field equations for the superconducting order parameter, going beyond the Suhl, Matthias and Walker (SMW) extension of the BCS theory 37. This results in an extended self-consistent and phase-dependent set of equations for the several components of the order parameter, with strongly hybridized excitation spectra. The meanfield Hamiltonian including both intraband and crossband pairing reads:

$$
\begin{aligned}
H & =\sum_{i, \mathbf{k}, \sigma} \epsilon_{i}(\mathbf{k}) c_{i, \mathbf{k} \sigma}^{\dagger} c_{i, \mathbf{k} \sigma}+H_{i n t}, \\
H_{i n t} & =\sum_{i, j} \sum_{\mathbf{k}}\left[\Delta_{i j}(\mathbf{k}) c_{i, \mathbf{k} \uparrow}^{\dagger} c_{j,-\mathbf{k} \downarrow}^{\dagger}+h . c .\right],
\end{aligned}
$$

where $i, j=1,2$ represent the band in$\operatorname{dex}$, and $\sigma=\uparrow, \downarrow$ the spin. Here $\Delta_{i j}(\mathbf{k})=$ $\sum_{k, l=1,2} \sum_{\mathbf{k}^{\prime}} V_{i j, k l}\left(\mathbf{k}, \mathbf{k}^{\prime}\right)\left\langle c_{k,-\mathbf{k} \uparrow} c_{l, \mathbf{k}^{\prime} \downarrow}\right\rangle$ are the pairing amplitudes, and $\epsilon_{i}(\mathbf{k})$ is the band-dependent kinetic energy of the electrons. We note that Eq. (1) resembles the Hamiltonian of a two-band system with hybridization upon the change from orbital to the band basis [31. The full $\mathbf{k}$-dependent form of the interaction matrix is given by $V_{i j, k l}\left(\mathbf{k}, \mathbf{k}^{\prime}\right)=-g_{i j, k l} \Theta\left(\Omega-\left|\zeta_{i}(\mathbf{k})\right|\right) \Theta(\Omega-$ $\left.\left|\zeta_{j}(\mathbf{k})\right|\right) \Theta\left(\Omega-\left|\zeta_{k}\left(\mathbf{k}^{\prime}\right)\right|\right) \Theta\left(\Omega-\left|\zeta_{l}\left(\mathbf{k}^{\prime}\right)\right|\right)$, where $\Omega$ is the average energy scale of the effective interaction, and $\zeta_{i}(\mathbf{k})=\epsilon_{i}(\mathbf{k})-\mu$ with chemical potential $\mu$. In $g_{i j, k l}=\left(\begin{array}{ccc}g_{11,11} & g_{11,22} & g_{11,(12)} \\ g_{22,11} & g_{22,22} & g_{22,(12)} \\ g_{(12), 11} & g_{(12), 22} & g_{(12),(12)}\end{array}\right)$, the upper left $2 \times 2$ inner matrix corresponds to the well established SMW case [37, and the third row and column include the crosspairing (where (12) indicates symmetrization under given indices, so that e.g., $\left.g_{(12),(12)}=g_{12,12}+g_{21,21}\right)$. In the interaction matrix the effective attraction between electrons is given by its diagonal elements, and the off-diagonal ones describe the Josephson-like coupling between intraband and cross-band condensates.

In what follows, we simplify our indices as $11 \equiv 1$, $22 \equiv 2$ and $(12) \equiv 3$. Next, we use the Gor'kov Green's functions formalism to obtain the pair amplitude equations [19, 38]. In momentum space the two excitation spectra without crosspairing $(i=1,2)$ are $\varepsilon_{i}^{2}=\zeta_{i}^{2}+\left|\Delta_{i}\right|^{2}$ and the pair amplitudes are given by $\Delta_{i}(\mathbf{k})=\left|\Delta_{i}\right| e^{i \varphi_{i}} \Theta\left(\Omega-\left|\zeta_{i}(\mathbf{k})\right|\right)$, where $\varphi_{i}$ is the phase of the pair amplitude.

The crosspairing pair amplitude $\Delta_{3}$ hybridizes the energy spectra of the two BCS-like excitation branches:

$$
\begin{aligned}
E_{ \pm}(\theta) & =\sqrt{\frac{1}{2}\left(\varepsilon_{1}^{2}+\varepsilon_{2}^{2}+2\left|\Delta_{3}\right|^{2} \pm b(\theta)\right)}, \\
b(\theta) & =\sqrt{\left(\varepsilon_{1}^{2}-\varepsilon_{2}^{2}\right)^{2}+4\left|\Delta_{3}\right|^{2} r(\theta),}
\end{aligned}
$$

where $r=\left(\zeta_{1}-\zeta_{2}\right)^{2}+\left|\Delta_{1}\right|^{2}+\left|\Delta_{2}\right|^{2}+2\left|\Delta_{1}\right|\left|\Delta_{2}\right| \cos \theta$ and $\theta=2 \varphi_{3}-\varphi_{1}-\varphi_{2}$. We emphasize here that the angle $\theta$ will introduce new degrees of freedom in our system depending on the combination of the couplings, as will be shown later. The excitation gaps $\Delta_{ \pm}(\theta)$ coincide with the minimum energy of the excitation branches $E_{ \pm}(\theta)$. These are the two gaps $\Delta_{ \pm}$present in the density of states (DOS), however these gaps no longer correspond to the energy needed to break intraband Cooper pairs (as is conventionally the case). Instead, they describe the energy needed to disallow either intra- or cross-band pairing.

The self-consistent equations for the pair amplitudes are given by:

$$
\Delta_{i}=\frac{1}{2} \sum_{j} g_{i j} \int \frac{d^{3} k}{(2 \pi)^{3}} \Delta_{j}\left[\chi_{j}^{+} f\left(E_{+}\right)+\chi_{j}^{-} f\left(E_{-}\right)\right],
$$

where $f(E)=\frac{1}{2 E} \tanh \left(\frac{\beta E}{2}\right), \chi_{i}^{ \pm}=1 \pm \frac{1}{b(\theta)} \chi_{i}, \chi_{1(2)}=$ $\varepsilon_{1(2)}^{2}-\varepsilon_{2(1)}^{2}+2\left|\Delta_{3}\right|^{2}\left(1+\left|\Delta_{2(1)}\right| e^{i \theta} /\left|\Delta_{1(2)}\right|\right), \quad \chi_{3}=$ $\left(\zeta_{1}-\zeta_{2}\right)^{2}+\left|\Delta_{1}\right|^{2}+\left|\Delta_{2}\right|^{2}+2\left|\Delta_{1}\right|\left|\Delta_{2}\right| e^{-i \theta}$, and $\beta=$ $1 / k_{B} T$. Note that these pairing amplitudes (i.e. the order parameters in the problem) do not correspond to the measurable gaps $\Delta_{ \pm}$.

Before solving the above formalism to reveal new physics brought by crosspairing, we introduce parabolic bands and dimensionless effective couplings, $\lambda_{i j}=$ $g_{i j} N_{j}(0)$, where $N_{j=1,2}(0)$ is the band-dependent density of states and $N_{3}(0)=N_{1}(0)+N_{2}(0)$. We start by solving Eq. (5) when all couplings $\lambda_{i j}$ are positive and with the same phase, i.e. $\theta=0$. To visualize the effect of crosspairing we fix all parameters but $\lambda_{33}: E_{F}=200 \mathrm{meV}$, $\Omega=30 \mathrm{meV}, \lambda_{11}=0.4, \lambda_{22}=0.3, \lambda_{i j, i \neq j}=0.05$. In Figs. 2(a) and 2(b), we show the excitation gaps and all three pairing amplitudes at $4.2 \mathrm{~K}$. As crosspairing coupling $\lambda_{33}$ is increased, the two excitation gaps $\Delta_{+}$and $\Delta_{-}$are split further apart: increasing $\Delta_{3}$ strengthens $\Delta_{+}$ and suppresses $\Delta_{-}$, up to a characteristic value $\lambda_{33}=\lambda_{c}$ (roughly half the average of $\lambda_{11}$ and $\lambda_{22}$ ). This characteristic value marks the maximal competition between the intraband and the crossband pairing channels and separates the two regimes: the intraband-dominated regime (IDR) for $\lambda_{33}<\lambda_{c}$, and a crosspairing-dominated regime (CDR) for $\lambda_{33}>\lambda_{c}$. In the CDR, both gaps increase at the same rate, similarly to the one-band scenario. Therefore the CDR describes a two-gap system which is characterized by a sole order parameter $\Delta_{3}$, while the intraband pair amplitudes participate only passively, by proximity [39, 40]. Fig. 2(c) shows that superconducting critical temperature $T_{c}$ increases with $\lambda_{33}$ faster than expected considering the range of values of $\lambda_{33}$ alone.

In the miniplots above Fig. 2(a), we show the density of states (as a measurable quantity in STM/STS) for the IDR, CDR as well as for the crossover point $\lambda_{33}=\lambda_{c}$. Note that in the latter situation the inner coherence peak approaches zero energy, and may disappear at exactly zero for a favorable combination of parame- 


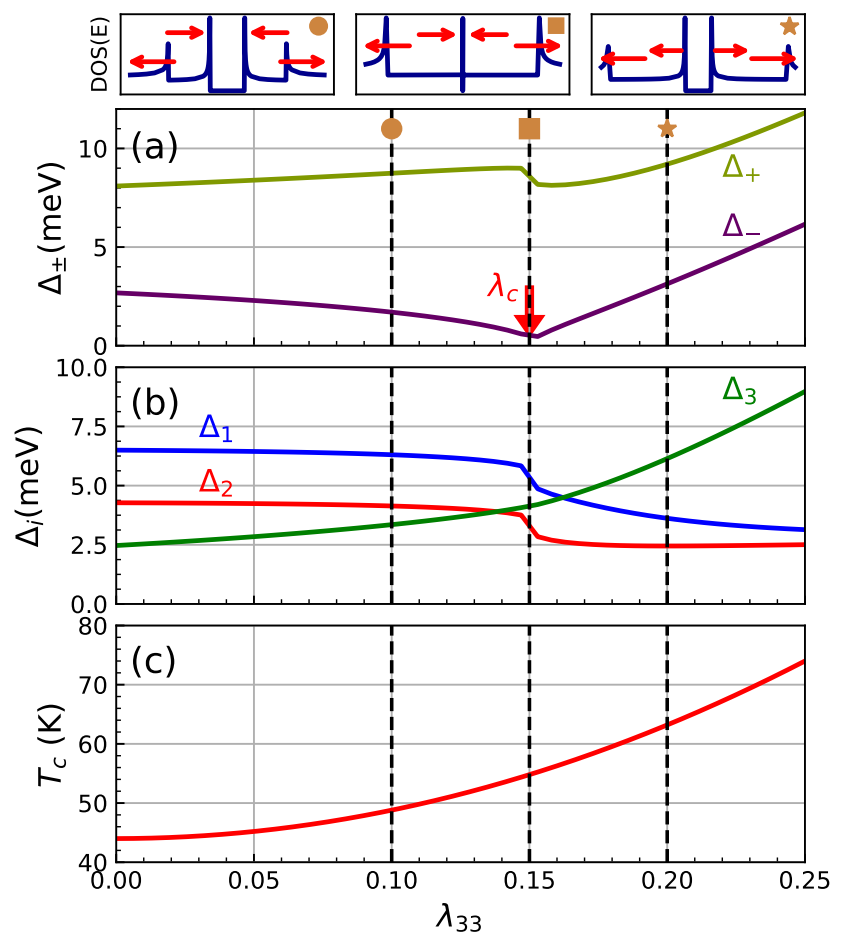

FIG. 2. Effect of crosspairing for the same phase. Excitation gaps (a) with their corresponding pair amplitudes (b) as function of $\lambda_{33}$ at $T=4.2 \mathrm{~K}$. The three miniplots above (a) show the density of states for $\lambda_{33}=0.1,0.15$, and 0.2 , illustrating the behavior in the intraband-dominated regime, gapless state, and the crosspairing dominated regime, respectively. (c) Mean-field critical temperature versus $\lambda_{33}$.

ters. That case would mark a gapless regime, where the weaker gap is no longer directly detectable, but must play a role in all observables in e.g., applied magnetic field or transport measurements. In such a state, superconducting gaps extracted from the tunneling spectra of STM would no longer coincide with the ones extracted from low-temperature ARPES [41] using normal-state band structure as a reference. Moreover, the lowest energy excitation branch exhibits linear V-shaped dispersion in the gapless state (see Fig. 3). Such a multiband system has a peculiar multicomponent composition, with the coexistence of a large-gap condensate and the in-gap states having a free-particle character. This leads to a finite DOS at low energies, and radically changed temperature dependence of all superconducting properties with respect to the gapped state. One concludes that such a gapless state, induced by crosspairing, is a unique feature of multiband superconductors worthy of further investigation.

To quantify the effects of crosspairing, it is instructive to take the example of the best known two-gap superconductor $\mathrm{MgB}_{2}$ [42. This superconductor has four contributing bands, two $\sigma$-bands for the stronger gap and two $\pi$-bands for the weaker one. The distance of two $\sigma$ -

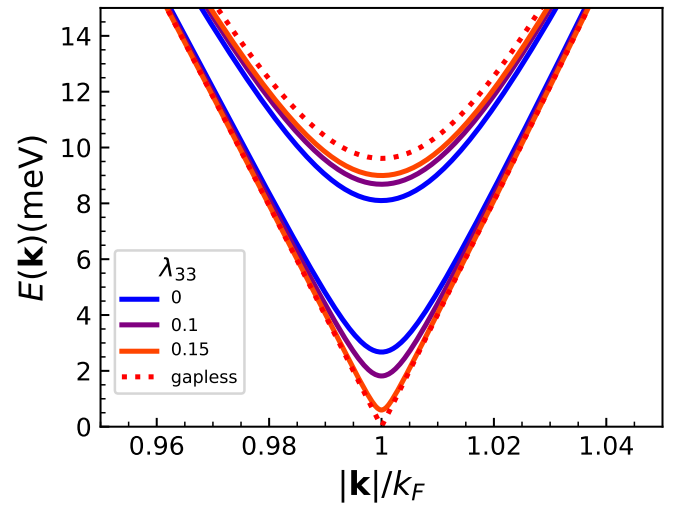

FIG. 3. Energy dispersions corresponding to Fig. 2 for $\lambda_{33}$ increased towards the gapless state.

bands in the vicinity of the Fermi level is approximately $75 \mathrm{meV}$ [see Fig. 1(a)]. Taking the parameters $\mu=500$ $\mathrm{meV}$ and $\Omega=75 \mathrm{meV}$ from Refs. 35, 43, we consider the crosspairing between the $\sigma$-bands, with the coupling matrix

$$
\lambda_{i j}=\left(\begin{array}{cccc}
0.275 & 0.032 & \lambda_{i 3} & 0.032 \\
0.032 & 0.274 & \lambda_{i 3} & 0.032 \\
\lambda_{i 3} & \lambda_{i 3} & 0.1 & 0.01 \\
0.01 & 0.01 & 0.01 & 0.22
\end{array}\right)
$$

The above matrix is asymmetric because of different DOS associated with each band. $\lambda_{i 4}$ is the coupling to the $\pi$ bands, and third column and row correspond to the coupling to the crosspairing channel, with $\lambda_{i 3}$ as a free (small) parameter. Other coupling constants are taken from literature, and yield the experimentally verified gaps of $\mathrm{MgB}_{2}(\approx 7$ and $3 \mathrm{meV})$ in absence of crosspairing $\left(\lambda_{i 3}=0\right.$, see Fig. 4). Even a small $\lambda_{i 3}=0.01$ yields a $2 \mathrm{meV}$ split of the two $\sigma$ gaps and a $1 \mathrm{~K}$ increase in $T_{c}$. This gives confidence that crosspairing effects, even if seemingly small, can lead to significant modifications of the gap spectrum without significantly changing $T_{c}$. That in turn calls for revisiting of theoretical approaches, e.g., to include crosspairing in anisotropic Eliashberg calculations even for materials that seemed previously well described [44, 45, as well as revisiting the available experimental data (bearing in mind the non-equivalence between $\Delta_{ \pm}$and the pairing amplitudes in presence of crosspairing). Conducting more refined ARPES measurements (e.g., in case of crystalline $\mathrm{MgB}_{2}$, on two $\sigma$ bands separately) can provide evidence for the gap splitting caused by crosspairing.

Last but not least, we discuss the phase-frustrated solutions of Eq. (5), with non-zero angle $\theta$. For example, in the family of FeSCs one can have two cases where a non trivial phase difference is present. The first is the conventional $s^{+-}$case, which contemplates a $\pi$-phase difference between electron-like and hole-like pair amplitudes [46. The second is the orbital antiphase $s^{+-}$case, 


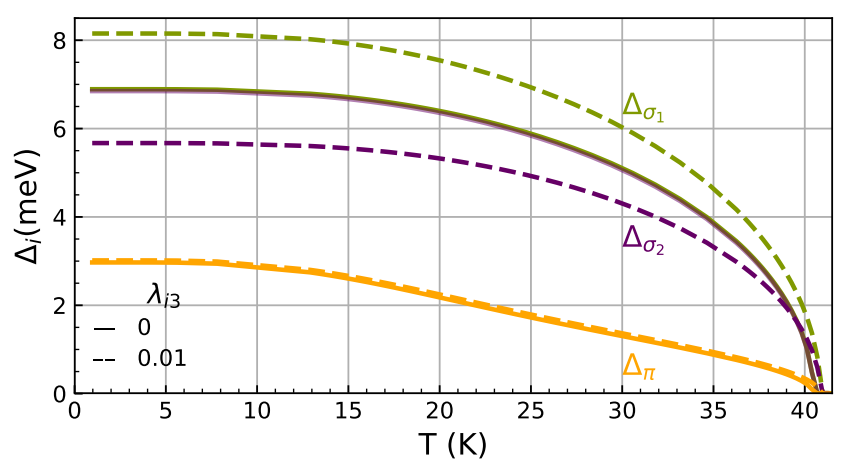

FIG. 4. Superconducting gaps of bulk $\mathrm{MgB}_{2}$ as a function of temperature, for intraband pairing only (solid lines), and in the presence of weak crosspairing (dashed lines).

with a $\pi$-phase difference between bands of the same type (electron-like or hole-like), as reported in the optimally doped $(\mathrm{BaK}) \mathrm{Fe}_{2} \mathrm{As}_{2}\left(T_{c}=36 \mathrm{~K}\right)$ [47 49]. This compound presents two hole-like bands $(\alpha, \beta)$ stemming from two nested Fermi sheets at $\Gamma$-point, and two electron-like bands $(\gamma, \delta)$ stemming from two nested Fermi sheets at the M-point. The proximity of both pairs of bands to the Fermi level and the smallness of their interband distance justifies the assumption of crosspairing between bands $\alpha$ and $\beta$ or $\gamma$ and $\delta$. To identify the emergent effects, we will consider the effect of crosspairing only between $\alpha$ and $\beta$ (assuming similar consequences for crosspairing between $\gamma$ and $\delta$ ). We take the interband distance between $\alpha$ and $\beta$ as $10 \mathrm{meV}$ and the Fermi level at $\mu=50 \mathrm{meV}$, following Ref. [50]. To obtain the gaps $\left(\Delta_{ \pm}\right)$as measured in low-temperature experiments of Ref. 16 ( $\approx 12.4$ and 6.2 meV extrapolated to $T=0$ ), we take for the coupling matrix:

$$
\lambda_{i j}=\left(\begin{array}{ccc}
0.51 & \lambda_{12} & \lambda_{13} \\
0.5 \lambda_{12} & 0.39 & \lambda_{13} \\
0.5 \lambda_{13} & 0.5 \lambda_{13} & 0.25
\end{array}\right)
$$

Here $\lambda_{12}$ is taken negative, which is the standard way to obtain the sign change in the band-dependent order parameters (as reported in $\mathrm{Ba}_{0.6} \mathrm{~K}_{0.4} \mathrm{Fe}_{2} \mathrm{As}_{2}$ [51]). We introduce a small repulsion $\lambda_{12}=-0.005$, which induces a phase shift between the two intraband pair amplitudes, $\varphi_{1}-\varphi_{2}=\pi$. In such a case, the coupling of the crosspairing pair amplitude with the intraband pair amplitudes (for $\lambda_{i 3}>0$ ) will introduce frustration on the phase of the crosspairing order parameter $\varphi_{3}$. Phase frustration of similar sort is known in three-band systems [52 54 . and can lead to skyrmionic vortex states [55 57, but is not possible in a two-band system unless crosspairing is present. In the present case, we reveal additional new physics, as crosspairing induces $s^{+-} \rightarrow s^{++}$transition as a function of temperature, as shown in Fig. $5(\mathrm{a}, \mathrm{b})$ for exemplified parameters of $(\mathrm{BaK}) \mathrm{Fe}_{2} \mathrm{As}_{2}$.

In the example shown in Fig. 5(a,b), after the transi-
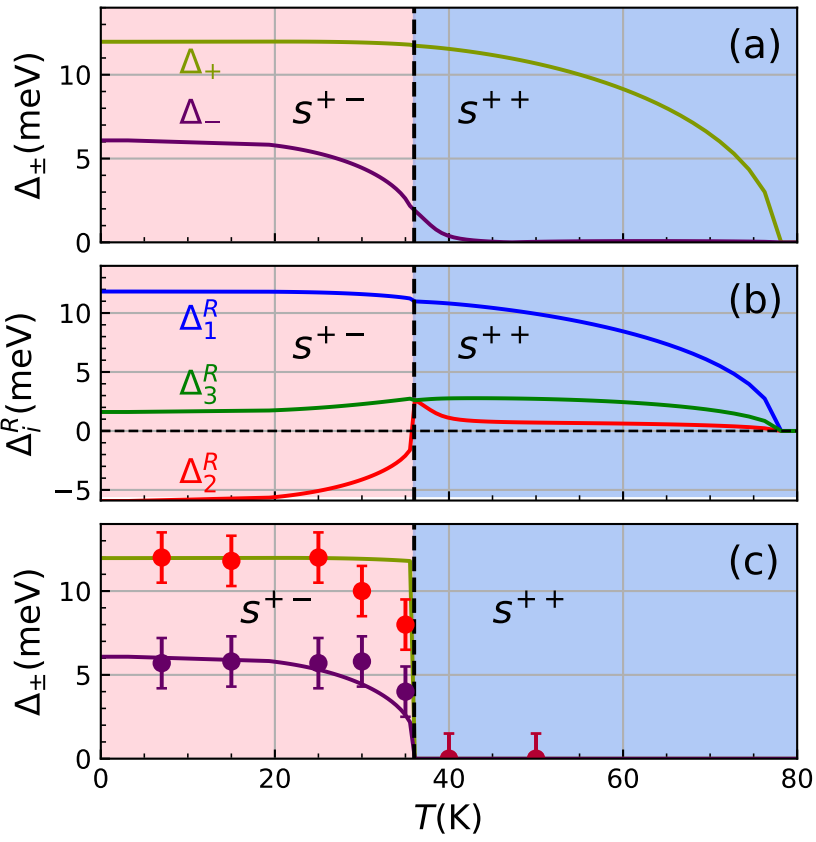

FIG. 5. (a) Excitation gaps $\Delta_{ \pm}$, and (b) real part of the pair amplitudes $\Delta_{i}^{R}$ as a function of temperature, for parameters of $(\alpha, \beta)$ bands in $(\mathrm{BaK}) \mathrm{Fe}_{2} \mathrm{As}_{2}$, with nominal $s^{+-}$antiphase and in presence of crosspairing. For parameters given in the text, $s^{+-} \rightarrow s^{++}$transition is found at $36 \mathrm{~K}$, corresponding to the critical temperature of the measured gaps at the $\Gamma$-point of $\mathrm{Ba}_{0.6} \mathrm{~K}_{0.4} \mathrm{Fe}_{2} \mathrm{As}_{2}$ [16]. Panel (c) superimposes the theoretical data of (a) on experimental data of Ref. [16], highlighting their agreement in case $s^{+-}$antiphase is protected.

tion, the pair amplitudes recover the same phase $(\theta=0)$ until the expected BCS critical temperature of $\approx 80 \mathrm{~K}$. In experiment however [16, the measured gaps abruptly cease at $T_{c} \approx 40 \mathrm{~K}$, for reasons that are not understood to date. Without claiming to rigorously describe the non BCS behavior of the gaps versus temperature, we notice that our calculation of the gaps vs. temperature can closely reproduce the experimentally measured data [as shown in Fig. [5(c)], in cases that the $s^{+-}$orbital antiphase is protected by symmetry or the transition to $s^{++}$state is disallowed in any way.

In summary, although mostly neglected to date, the cross-band pairing in multiband superconductors is certainly of importance in materials with hybridized or energetically close bands in the vicinity of the Fermi level. In this regime, the interplay between intra- and cross-band pairing leads to several unique effects. For one, crossband pairing increases the splitting between intraband gaps, with a tendency to decrease the weaker gap towards an entirely novel "gapless" state, signatures of which will still be observable since vanishing gap does not imply vanishing order parameter(s) in this regime. The crosspairing also introduces the possibility of a phase frustration between the pairing channels, leading to novel transi- 
tions as a function of temperature ( $\operatorname{such}$ as $s^{+-} \rightarrow s^{++}$), and likely nontrivial response of the superconductor to e.g., magnetic field 58. Our results call for revisiting the existing theories and experimental data for multiband superconductors with close bands, bearing also in mind that the band dispersions and chemical potential can be tuned towards a parameter regime where the above mentioned signatures of crosspairing can be detected. In that context, we point out the most recent measurements of Ref. 4, where tunability of multiple gaps has been achieved at the oxides' interface by gate doping around a Lifshitz transition, as the closest experimental system to our present model.

Besides the needed generalization to the case of multiple $(3+)$ bands, the outlook of the present study is very broad. It includes understanding the effects of impurities, particularly magnetic ones where DOS signatures of crosspairing near a gapless state can overlap with the Majorana zero-energy bound state [59,60]. It is also of interest to further examine the intra- to cross-pairing competition in the presence of spin-flip scattering [61, oddness in parity [15, and photo-induced phenomena [9, 62. Even beyond superconductivity, crosspairing and its competition with intraband pairing remains insufficiently explored in molecular optics 63], multicomponent superfluidity [28], and quantum chromodynamics 29].

The authors thank Andrea Guidini for his help during the initial stage of this work and Laura Fanfarillo for useful discussions. This work was partially supported by the Italian MIUR through the PRIN 2015 program (contract No. 2015C5SEJJ001) and the Research Foundation - Flanders (FWO). A. A. Vargas-Paredes acknowledges support by the joint doctoral program "MultiSuper" (http://www.multisuper.org) and by the Erasmus+ exchange and M.V. Milošević acknowledges the University of Camerino for support during his visit.

[1] M. V. Milošević and A. Perali, "Emergent phenomena in multicomponent superconductivity: an introduction to the focus issue," Supercond. Sci. Technol. 28, 060201 (2015)

[2] F. Giorgianni, T. Cea, C. Vicario, C. P. Hauri, W. K. Withanage, X. Xi, and L. Benfatto, "Leggett mode controlled by light pulses," Nature Physics 15, 341-346 (2019)

[3] T. Hanaguri, S. Kasahara, J. Böker, I. Eremin, T. Shibauchi, and Y. Matsuda, "Quantum Vortex Core and Missing Pseudogap in the Multiband BCS-BEC Crossover Superconductor FeSe," Phys. Rev. Lett. 122, 077001 (2019)

[4] G. Singh, A. Jouan, G. Herranz, M. Scigaj, F. Sánchez, L. Benfatto, S. Caprara, M. Grilli, G. Saiz, F. Couëdo, C. Feuillet-Palma, J. Lesueur, and N. Bergeal, "Gap suppression at a lifshitz transition in a multicondensate superconductor," Nature Materials (2019),
$10.1038 / \mathrm{s} 41563-019-0354-\mathrm{Z}$

[5] Y. Li, C. An, C. Hua, X. Chen, Y. Zhou, Y. Zhou, R. Zhang, C. Park, Z. Wang, Y. Lu, Y. Zheng, Z. Yang, and $\mathrm{Z}$. $\mathrm{Xu}$, "Pressure-induced superconductivity in topological semimetal NbAs2," npj Quantum Materials 3, 58 (2018)

[6] W.X. Li, R. Zeng, L. Lu, and S.X. Dou, "Dependence of superconducting properties on lattice strain in $\mathrm{MgB}_{2}$," Physica C: Superconductivity and its Applications 470, S629 - S630 (2010), proceedings of the 9th International Conference on Materials and Mechanisms of Superconductivity.

[7] D. Costanzo, S. Jo, H. Berger, and A. F. Morpurgo, "Gate-induced superconductivity in atomically thin MoS2 crystals," Nature Nanotechnology 11, 339 (2016)

[8] A. Continenza and G. Profeta, "Chemical doping in pnictides superconductors: The case of $\mathrm{Ca}\left(\mathrm{Fe}_{1-x} \mathrm{X}_{x}\right)_{2} \mathrm{As}_{2}, \mathrm{X}$ $=\mathrm{Co}, \mathrm{Ni}, \mathrm{Pt}$," Journal of Magnetism and Magnetic Materials 452, 179 - 183 (2018).

[9] S. Porta, L. Privitera, N. Traverso Ziani, M. Sassetti, F. Cavaliere, and B. Trauzettel, "Feasible model for photo-induced interband pairing," arXiv:1903.12396 [cond-mat.mes-hall].

[10] J. Nagamatsu, N. Nagakawa, T. Muranaka, Y. Zenitani, and J. Akimitsu, "Superconductivity at $39 \mathrm{k}$ in magnesium diboride," Nature 410, 63 (2001).

[11] L. de' Medici, G. Giovannetti, and M. Capone, "Selective Mott Physics as a Key to Iron Superconductors," Phys. Rev. Lett. 112, 177001 (2014).

[12] L. Benfatto, B. Valenzuela, and L. Fanfarillo, "Nematic pairing from orbital-selective spin fluctuations in FeSe," npj Quantum Materials 3, 56 (2018)

[13] R. Yu, J. Zhu, and Q. Si, "Orbital Selectivity Enhanced by Nematic Order in FeSe," Phys. Rev. Lett. 121, 227003 (2018)

[14] J. Herbrych, N. Kaushal, A. Nocera, G. Alvarez, A. Moreo, and E. Dagotto, "Spin dynamics of the block orbital-selective Mott phase," Nature Communications 9, 3736 (2018).

[15] A. M. Black-Schaffer and A. V. Balatsky, "Odd-frequency superconducting pairing in multiband superconductors," Phys. Rev. B 88, 104514 (2013).

[16] H. Ding, P. Richard, K. Nakayama, K. Sugawara, T. Arakane, Y. Sekiba, A. Takayama, S. Souma, T. Sato, T. Takahashi, Z. Wang, X. Dai, Z. Fang, G. F. Chen, J. L. Luo, and N. L. Wang, "Observation of Fermi-surface-dependent nodeless superconducting gaps in $\mathrm{Ba}_{0.6} \mathrm{~K}_{0.4} \mathrm{Fe}_{2} \mathrm{As}_{2}$," EPL 83, 47001 (2008)

[17] A. Bianconi, A. Valletta, A. Perali, and N.L. Saini, "High tc superconductivity in a superlattice of quantum stripes," Solid State Communications 102, $369-$ 374 (1997)

[18] A. Valletta, A. Bianconi, A. Perali, and N.L. Saini, "Electronic and superconducting properties of a superlattice of quantum stripes at the atomic limit," Zeitschrift für Physik B Condensed Matter 104, 707-713 (1997)

[19] A. A. Shanenko, J. Albino Aguiar, A. Vagov, M. D. Croitoru, and M. V. Milošević, "Atomically flat superconducting nanofilms: multiband properties and meanfield theory," Supercond. Sci. Technol. 28, 054001 (2015).

[20] L. Flammia, L.-F. Zhang, L. Covaci, A. Perali, and M. V. Milošević, "Superconducting nanoribbon with a constriction: A quantum-confined josephson junction," 
Phys. Rev. B 97, 134514 (2018)

[21] L.-F. Zhang, L. Flammia, L. Covaci, A. Perali, and M. V. Milošević, "Multifaceted impact of a surface step on superconductivity in atomically thin films," Phys. Rev. B 96, 104509 (2017).

[22] D. Valentinis, S. Gariglio, A. Fête, J.-M. Triscone, C. Berthod, and D. van der Marel, "Modulation of the superconducting critical temperature due to quantum confinement at the $\mathrm{LaAlO}_{3} / \mathrm{SrTiO}_{3}$ interface," Phys. Rev. B 96, 094518 (2017).

[23] N. Mohanta and A. Taraphder, "Multiband theory of superconductivity at the $\mathrm{LaAlO}_{3} / \mathrm{SrTiO}_{3}$ interface," Phys. Rev. B 92, 174531 (2015).

[24] T. V. Trevisan, M. Schütt, and R. M. Fernandes, "Unconventional Multiband Superconductivity in Bulk $\mathrm{SrTiO}_{3}$ and $\mathrm{LaAlO}_{3} / \mathrm{SrTiO}_{3}$ Interfaces," Phys. Rev. Lett. 121, 127002 (2018).

[25] R.-S. Wang, Y. Gao, Z.-B. Huang, and X.-J. Chen, "Superconductivity above 120 kelvin in a chain link molecule," arXiv e-prints , arXiv:1703.06641 (2017), arXiv:1703.06641 [cond-mat.supr-con]

[26] X. Zhang, Y. Zhou, B. Cui, M. Zhao, and F. Liu, "Theoretical Discovery of a Superconducting Two-Dimensional Metal-Organic Framework," Nano Letters 17, 6166-6170 (2017)

[27] M. V. Mazziotti, A. Valletta, G. Campi, D. Innocenti, A. Perali, and A. Bianconi, "Possible fano resonance for high-tc multi-gap superconductivity in p-terphenyl doped by $\mathrm{k}$ at the lifshitz transition," EPL 118, 37003 (2017)

[28] W. V. Liu and F. Wilczek, "Interior gap superfluidity," Phys. Rev. Lett. 90, 047002 (2003).

[29] E. Gubankova, W. V. Liu, and F. Wilczek, "Breached Pairing Superfluidity: Possible Realization in QCD," Phys. Rev. Lett. 91, 032001 (2003).

[30] A. Moreo, M. Daghofer, J. A. Riera, and E. Dagotto, "Properties of a two-orbital model for oxypnictide superconductors: Magnetic order, $B_{2 \mathrm{~g}}$ spin-singlet pairing channel, and its nodal structure," Phys. Rev. B 79, $134502(2009)$

[31] A. Moreo, M. Daghofer, A. Nicholson, and E. Dagotto, "Interband pairing in multiorbital systems," Phys. Rev. B 80, 104507 (2009)

[32] C. E. Matt, D. Sutter, A. M. Cook, Y. Sassa, M. Månsson, O. Tjernberg, L. Das, M. Horio, D. Destraz, C. G. Fatuzzo, K. Hauser, M. Shi, M. Kobayashi, V. N. Strocov, T. Schmitt, P. Dudin, M. Hoesch, S. Pyon, T. Takayama, H. Takagi, O. J. Lipscombe, S. M. Hayden, T. Kurosawa, N. Momono, M. Oda, T. Neupert, and J. Chang, "Direct observation of orbital hybridisation in a cuprate superconductor," Nature Communications 9, $972(2018)$

[33] J. Tahir-Kheli, "Interband pairing theory of superconductivity," Phys. Rev. B 58, 12307-12322 (1998)

[34] O.V. Dolgov, E.P. Fetisov, and D.I. Khomskii, "Superconductivity of heavy fermions in a two-band model," Physics Letters A 125, 267 - 270 (1987).

[35] S. Ponc, E.R. Margine, C. Verdi, and F. Giustino, "Epw: Electronphonon coupling, transport and superconducting properties using maximally localized wannier functions," Computer Physics Communications 209, 116 133 (2016)

[36] J. Bekaert, L. Bignardi, A. Aperis, P. van Abswoude, C. Mattevi, S. Gorovikov, L. Petaccia, A. Goldoni,
B. Partoens, P. M. Oppeneer, F. M. Peeters, M. V. Milošević, P. Rudolf, and C. Cepek, "Free surfaces recast superconductivity in few-monolayer $\mathrm{MgB}_{2}$ : Combined first-principles and ARPES demonstration," Scientific Reports 7, 14458 (2017)

[37] H. Suhl, B. T. Matthias, and L. R. Walker, "BardeenCooper-Schrieffer Theory of Superconductivity in the Case of Overlapping Bands," Phys. Rev. Lett. 3, 552554 (1959)

[38] F. G. Korchorbé and M. E. Palistrant, "Superconductivity in a two-band system with low carrier density," Zh. Eksp. Teor. Fiz. 104, 442-451 (1993).

[39] F. Giubileo, F. Bobba, A. Scarfato, A. M. Cucolo, A. Kohen, D. Roditchev, N. D. Zhigadlo, and J. Karpinski, "Local tunneling study of three-dimensional order parameter in the $\pi$ band of Al-doped $\mathrm{MgB}_{2}$ single crystals," Phys. Rev. B 76, 024507 (2007).

[40] F. Giubileo, D. Roditchev, W. Sacks, R. Lamy, D. X. Thanh, J. Klein, S. Miraglia, D. Fruchart, J. Marcus, and Ph. Monod, "Two-Gap State Density in $\mathrm{MgB}_{2}$ : A True Bulk Property Or A Proximity Effect?" Phys. Rev. Lett. 87, 177008 (2001).

[41] Andrea Damascelli, Zahid Hussain, and Zhi-Xun Shen, "Angle-resolved photoemission studies of the cuprate superconductors," Rev. Mod. Phys. 75, 473-541 (2003).

[42] S. Souma, Y. Machida, T. Sato, T. Takahashi, H. Matsui, S.-C. Wang, H. Ding, A. Kaminski, J. C. Campuzano, S. Sasaki, and K. Kadowaki, "The origin of multiple superconducting gaps in $\mathrm{MgB}_{2}$," Nature 423, 65 (2003)

[43] S. A. Kuzmichev, T. E. Kuzmicheva, and S. N. Tchesnokov, "Determination of the electron-phonon coupling constants from the experimental temperature dependences of superconducting gaps in $\mathrm{MgB}_{2}$," JETP Letters 99, 295-302 (2014)

[44] H. J. Choi, D. Roundy, H. Sun, M. L. Cohen, and S. G. Louie, "First-principles calculation of the superconducting transition in $\mathrm{MgB}_{2}$ within the anisotropic Eliashberg formalism," Phys. Rev. B 66, 020513 (2002).

[45] A. Aperis, P. Maldonado, and P. M. Oppeneer, "Ab initio theory of magnetic-field-induced odd-frequency twoband superconductivity in $\mathrm{MgB}_{2}$," Phys. Rev. B 92, $054516(2015)$

[46] I. I. Mazin, D. J. Singh, M. D. Johannes, and M. H. Du, "Unconventional Superconductivity with a Sign Reversal in the Order Parameter of $\mathrm{LaFeAsO}_{1-x} \mathrm{~F}_{x}$," Phys. Rev. Lett. 101, 057003 (2008)

[47] X. Lu, C. Fang, W-F Tsai, Y. Jiang, and J. Hu, " $s$-wave superconductivity with orbital-dependent sign change in checkerboard models of iron-based superconductors," Phys. Rev. B 85, 054505 (2012)

[48] P. Zhang, P. Richard, T. Qian, X. Shi, J. Ma, L.-K. Zeng, X.-P. Wang, E. Rienks, C.-L. Zhang, P. Dai, Y.-Z. You, Z.-Y. Weng, X.-X. Wu, J. P. Hu, and H. Ding, "Observation of Momentum-Confined In-Gap Impurity State in $\mathrm{Ba}_{0.6} \mathrm{~K}_{0.4} \mathrm{Fe}_{2} \mathrm{As}_{2}$ : Evidence for Antiphase $\mathrm{s}_{ \pm}$Pairing," Phys. Rev. X 4, 031001 (2014)

[49] Z. P. Yin, K. Haule, and G. Kotliar, "Spin dynamics and orbital-antiphase pairing symmetry in iron-based superconductors," Nature Physics 10, 845 (2014)

[50] H. Ding, K. Nakayama, P. Richard, S. Souma, T. Sato, T. Takahashi, M. Neupane, Y-M Xu, Z-H Pan, A. V. Fedorov, Z. Wang, X. Dai, Z. Fang, G. F. Chen, J. L. Luo, and N. L. Wang, "Electronic structure of optimally doped pnictide $\mathrm{Ba}_{0.6} \mathrm{~K}_{0.4} \mathrm{Fe}_{2} \mathrm{As}_{2}$ : a comprehen- 
sive angle-resolved photoemission spectroscopy investigation," Journal of Physics: Condensed Matter 23, 135701 (2011)

[51] N. W. Salovich, H. Kim, A. K. Ghosh, R. W. Giannetta, W. Kwok, U. Welp, B. Shen, S. Zhu, H.-H. Wen, M. A. Tanatar, and R. Prozorov, "Effect of heavy-ion irradiation on superconductivity in $\mathrm{Ba}_{0.6} \mathrm{~K}_{0.4} \mathrm{Fe}_{2} \mathrm{As}_{2}$," Phys. Rev. B 87, 180502 (2013)

[52] V. G. Stanev and A. E. Koshelev, "Anomalous proximity effects at the interface of $s$ and $s_{ \pm}$superconductors," Phys. Rev. B 86, 174515 (2012)

[53] N. V. Orlova, A. A. Shanenko, M. V. Milošević, F. M. Peeters, A. V. Vagov, and V. M. Axt, "Ginzburglandau theory for multiband superconductors: Microscopic derivation," Phys. Rev. B 87, 134510 (2013).

[54] J. W. Brendan and P. D. Mukunda, "Time-reversalsymmetry-broken state in the BCS formalism for a multiband superconductor," J. Phys. Condens. Matter 25, $425702(2013)$

[55] J. Garaud, J. Carlström, and E. Babaev, "Topological solitons in three-band superconductors with broken time reversal symmetry," Phys. Rev. Lett. 107, 197001 (2011).

[56] J. Garaud, J. Carlström, E. Babaev, and M. Speight, "Chiral $\mathbb{C} P^{2}$ skyrmions in three-band superconductors," Phys. Rev. B 87, 014507 (2013)

[57] N. V. Orlova, P. Kuopanportti, and M. V. Milošević, "Skyrmionic vortex lattices in coherently coupled threecomponent bose-einstein condensates," Phys. Rev. A 94,
$023617(2016)$

[58] J. Garaud, M. Silaev, and E. Babaev, "Change of the vortex core structure in two-band superconductors at the impurity-scattering-driven $s_{ \pm} / s_{++}$crossover," Phys. Rev. B 96, 140503 (2017).

[59] D. Wang, L. Kong, P. Fan, H. Chen, S. Zhu, W. Liu, L. Cao, Y. Sun, S. Du, J. Schneeloch, R. Zhong, G. Gu, L. Fu, H. Ding, and H-J Gao, "Evidence for majorana bound states in an iron-based superconductor," Science 362, 333-335 (2018)

[60] J-X. Yin, Z. Wu, J-H. Wang, Z-Y. Ye, Jing Gong, X-Y. Hou, Lei Shan, Ang Li, X-J. Liang, X-X. Wu, Jian Li, C-S. Ting, Z-Q. Wang, J-P. Hu, P-H. Hor, H. Ding, and S. H. Pan, "Observation of a robust zero-energy bound state in iron-based superconductor $\mathrm{Fe}(\mathrm{Te}, \mathrm{Se})$," Nature Physics 11, 543 (2015).

[61] R. S. Gonnelli, D. Daghero, G. A. Ummarino, A. Calzolari, M. Tortello, V. A. Stepanov, N. D. Zhigadlo, K. Rogacki, J. Karpinski, F. Bernardini, and S. Massidda, "Effect of Magnetic Impurities in a Two-Band Superconductor: A Point-Contact Study of Mn-Substituted $\mathrm{MgB}_{2}$ Single Crystals," Phys. Rev. Lett. 97, 037001 (2006).

[62] N. Kumar and K. P. Sinha, "Possibility of photoinduced superconductivity," Phys. Rev. 174, 482-488 (1968).

[63] J. R. Abo-Shaeer, D. E. Miller, J. K. Chin, K. Xu, T. Mukaiyama, and W. Ketterle, "Coherent molecular optics using ultracold sodium dimers," Phys. Rev. Lett. 94, 040405 (2005). 